\title{
Behaviour of Crohn's disease according to the Vienna classification: changing pattern over the course of the disease
}

\author{
E Louis, A Collard, A F Oger, E Degroote, F Aboul Nasr El Yafi, J Belaiche
}

\begin{abstract}
Background-Crohn's disease is a heterogeneous disorder with both a genetic and environmental aetiology. Clinical classifications of the disease, such as the newly proposed Vienna classification, may help to define subgroups of patients suitable for studying the influence of specific genetic or environmental factors.
\end{abstract}

Aim-To assess the stability over the course of the disease of its location and behaviour, as determined according to the Vienna classification.

Patients and methods-The notes of 297 Crohn's disease patients regularly followed up at our institution were carefully reviewed retrospectively. The behaviour and location of the disease according to the Vienna classification were determined at diagnosis and after $1,3,5,10,15,20$, and 25 years of follow up. The proportions of the different behaviours and locations of the disease were calculated at these time points. A statistical analysis of the evolution of these characteristics over 10 years was performed on a subgroup of 125 patients with at least 10 years of follow up. The influence of age at diagnosis on location and behaviour of the disease was assessed as well as the influence of location on the behaviour of the disease.

Results-The location of the disease remained relatively stable over the course of the disease. Although the proportion of patients who had a change in disease location became statistically significant after five years $(p=0.01)$, over 10 years only $15.9 \%$ of patients had a change in location $(p<0.001)$. We observed a more rapid and prominent change in disease behaviour, which was already statistically significant after one year $(p=0.04)$. Over 10 years, $45.9 \%$ of patients had a change in disease behaviour $(p<0.0001)$. The most prominent change was from non-stricturing non-penetrating disease to either stricturing $(27.1 \% ; \quad \mathrm{p}<0.0001)$ or penetrating $(29.4 \% ; \mathrm{p}<0.0001)$ disease. Age at diagnosis had no influence on either location or behaviour of disease. Ileal Crohn's disease was more often stricturing, and colonic or ileocolonic Crohn's disease was more often penetrating: this was already the case at diagnosis and became more prominent after 10 years $(p<0.05)$.
Conclusions-Location of Crohn's disease, as defined by the Vienna classification, is a relatively stable phenotype which seems suitable for phenotypegenotype analyses. Behaviour of Crohn's disease according to the Vienna classification varies dramatically over the course of the disease and cannot be used in phenotype-genotype analyses. The potential influence of genes on the behaviour of Crohn's disease should be studied in subgroups of patients defined by their disease behaviour after a fixed duration of disease.

(Gut 2001;49:777-782)

Keywords: Crohn's disease; genetics; Vienna classification

Crohn's disease is a multifactorial polygenic disease with probable genetic heterogeneity. ${ }^{1}$ In this hypothesis, different genetic backgrounds may explain different clinical patterns of the disease. ${ }^{2}$ Thus apart from specific disease predisposing genes, such as the recently described Nod $2,3^{4}$ some disease modifying genes may also exist. Various disease phenotypes that may be genetically determined have been suggested. ${ }^{5}$ These include age at diagnosis, sex, family history, location of disease, response to treatment, and what has been called behaviour of disease. To date few data, largely uncorroborated, have been published on the genetics of these phenotypes. ${ }^{6-9}$ This may be due to the complexity of the genetic model and to the large number of possible candidate genes for the various characteristics of the disease as well as interference from environmental influences. However, the main problem with these studies may be the irrelevance of the phenotypes studied. To be considered possibly genetically determined, a phenotype should be stable over time. Obviously this is the case for age at diagnosis and sex, but for the other characteristics, in particular location and behaviour of disease, stability of the phenotypes remains to be proved.

Behaviour of disease first referred to the indication for surgery: either perforating or non-perforating disease. ${ }^{10}$ These definitions were extended to non-surgical situations and behaviour was then termed as: primarily penetrating, primarily fibrostenotic, or primarily inflammatory, depending on the dominating feature at a given point in time. ${ }^{11}$ However, penetrating and fibrostenotic features often
Accepted for publicatic 3 July 2001 
coexist and this classification proved to be difficult to reproduce. ${ }^{12}$ A new classification based on disease behaviour was then proposed at the 1998 world gastroenterology meeting in Vienna. ${ }^{13}$ In this classification system, a hierarchy was introduced for stricturing and penetrating complications to try and improve reproducibility. Some arguments exist for a fixed disease behaviour in individual patients, including indications for surgery ${ }^{10-16}$ and relative conservation of disease behaviour in multiply affected families. ${ }^{17-19}$ However, the stability of disease behaviour over the course of the disease has never been studied specifically using a standardised classification.

Location of disease is another characteristic that could be genetically determined, at least in part. In common with disease behaviour, some conservation of this character has been described in multiply affected families. ${ }^{17-19}$ Furthermore, specific disease locations have been linked to serological or genetic markers such as antineutrophil antibodies. ${ }^{20}{ }^{21}$ Again, the Vienna classification system has proposed some modifications in an attempt to produce more relevant and stable categories. ${ }^{13}$ Furthermore, as it was considered that intestinal resection would significantly interfere with the spontaneous location of the disease, characterisation of disease location was decided before the first surgical procedure. As for disease behaviour, it remains unclear whether these newly defined phenotypes represent stable phenotypes suitable for genotype-phenotype association studies.

Therefore, the aim of our work was to assess the evolution over time of behaviour and location of Crohn's disease, as defined by the Vienna classification. In particular, we wished to examine stable characters that may be relevant for genetic studies.

\section{Patients and methods}

CLASSIFICATION OF PATIENTS

From a computed database of 500 patients with Crohn's disease seen at our university hospital, we selected 297 patients who were regularly followed up in our gastroenterology department. We then reviewed the medical notes of these 297 patients. For each patient, we determined current A (age at diagnosis), L (location), and B (behaviour) status of the Vienna classification ${ }^{13}$ (table 1 ). We then retrospectively determined LB status at diagnosis, and after $1,3,5,10,15,20$, and 25 years of evolution.

According to the Vienna classification, patients were defined as A1 if the diagnosis was made before 40 years of age and A2 if it was made thereafter. L1 corresponds to disease

Table 1 The Vienna classification of Crohn's disease

\begin{tabular}{ll}
\hline Age at diagnosis & A1, <40 years \\
& A2, >40 years \\
Location & L1, Terminal ileum \\
& L2, Colon \\
& L3, Ileocolon \\
& L4, Upper gastrointestinal \\
Behaviour & B1, non-stricturing non-penetrating \\
& B2, Stricturing \\
& B3, Penetrating \\
\hline
\end{tabular}

located in the terminal ileum (possibly involving the caecum), L2 in the colon, L3 ileocolon, and L4 involving the upper gastrointestinal tract (irrespective of the other locations of the disease). Location of disease is defined by the maximal extent of the disease but in case of surgical resection during the course of the disease, it is determined before the first resection. B1 corresponds to a non-stricturing nonpenetrating disease, B2 stricturing, and B3 penetrating. A disease is classified as B3 as soon as there are intra-abdominal or perianal fistula, perianal ulcer, inflammatory mass, and/or abscess at any time during the course of the disease, even if there are coexisting strictures. According to this classification, unlike that for location, the behaviour category of the disease was not decided after the first surgery and no specific limit during the course of the disease was imposed to define it. We also classified patients for behaviour and location depending on age classification and for behaviour depending on location.

Age at diagnosis was usually easy to find in the medical notes of the patients. For location of disease, we used $x$ ray and endoscopy protocols as well as surgical reports. For disease behaviour, we used $x$ ray, computed tomography scan, magnetic resonance imaging, ultrasound, and endoscopy protocols as well as proctology and surgical reports. When data were missing or unclear, the item was unclassified until more recent data were available.

EXPRESSION OF RESULTS AND STATISTICS

In the description of our population according to the Vienna classification criteria at the last visit, the proportion of patients in the various categories as well as the percentage of unclassified patients are given. In all other results the percentage of unclassified patients are no longer given and the proportions are calculated on the number of classified patients to avoid the influence of the number of unclassified patients on the percentages observed in the various categories. There were few data missing at the different time points, never exceeding $3 \%$ of patients.

To check for a statistically significant trend in disease behaviour or change in location, we tested the hypothesis of a proportion of change different from 0 between diagnosis and 10 years of evolution in a subgroup of 125 patients with a follow up of at least 10 years. The proportion was considered statistically significant when the ratio between the observed proportion and standard error was greater than $1.96(\mathrm{p}<0.05)$.

In the description of disease behaviour and change in location over 10 years in the 125 patients, duration before change is given as median (range).

When we studied the influence of age at diagnosis on behaviour and location of disease, as well as the influence of location on behaviour of disease, we compared percentages of patients in the various categories using Fisher's exact test with a level of significance set at $\mathrm{p}<0.05$. 
Table 2 Classification B (behaviour) and L (location) (\%) at diagnosis and at different time points in the course of the disease (years)

\begin{tabular}{lllllllll}
\hline \multicolumn{7}{c}{ Disease duration $(y)$} \\
\cline { 2 - 9 } & $\begin{array}{l}1 \\
(n=297)\end{array}$ & $\begin{array}{l}1 \\
(n=259)\end{array}$ & $\begin{array}{l}3 \\
(n=218)\end{array}$ & $\begin{array}{l}5 \\
(n=187)\end{array}$ & $\begin{array}{l}10 \\
(n=125)\end{array}$ & $\begin{array}{l}15 \\
(n=74)\end{array}$ & $\begin{array}{l}20 \\
(n=47)\end{array}$ & $\begin{array}{l}25 \\
(n=32)\end{array}$ \\
\hline B1 & 73.7 & 67.1 & 61.0 & 52.0 & 30.6 & 22.2 & 19.1 & 12.5 \\
B2 & 10.8 & 13.3 & 14.8 & 21.2 & 32.2 & 34.7 & 32 & 31.3 \\
B3 & 15.5 & 19.7 & 24.3 & 26.8 & 37.2 & 43.1 & 48.8 & 56.3 \\
L1 & 44.8 & 45.6 & 46.0 & 45.1 & 43.3 & 44.3 & 48.9 & 48.4 \\
L2 & 26.7 & 26.6 & 27.0 & 25.3 & 23.3 & 22.9 & 20.0 & 12.9 \\
L3 & 24.2 & 23.8 & 25.1 & 28.0 & 30.0 & 28.6 & 26.7 & 35.5 \\
L4 & 4.3 & 4.0 & 1.9 & 1.6 & 3.3 & 4.3 & 4.4 & 3.2 \\
\hline
\end{tabular}

\section{Results}

ABL CLASSIFICATIONS AT THE LAST VISIT

We had recent clinical data (within the last two years) for 264 of the 297 patients studied. Median follow up at the last visit for these patients was 9 years ( 2 months- 34 years). The proportions of patients in the different classifications according to items $\mathrm{A}, \mathrm{L}$, and $\mathrm{B}$ of the Vienna system were as follows: A1, 83.5\%; A2, $16.5 \%$; L1, 37.5\%; L2, 21.6\%; L3, 32.9\%; L4, $5.7 \% ; \mathrm{L}$ ?, $2.3 \% ; \mathrm{B} 1,28 \% ; \mathrm{B} 2,27.7 \% ; \mathrm{B} 3$, $43.2 \%$; B?, $1.1 \%$.

BL CLASSIFICATIONS AT DIAGNOSIS AND AT DIFFERENT TIME POINTS IN THE COURSE OF THE DISEASE

We had clinical data for classification at diagnosis for all 297 patients. The numbers of patients available for classification at $1,3,5,10$, 15,20 , and 25 years over the course of the disease were $259,218,187,125,74,47$, and 25 , respectively. Evolution over time of the proportion of patients classified according to items $\mathrm{L}$ and $\mathrm{B}$ of the Vienna classification are shown in table 2. There was a dramatic change in the proportion of various disease behaviours while the change in distribution of disease location was minimal. When examining the rate of change in behaviour over time, and particularly the rate of appearance of complicated forms, we found $26.3 \%$ of patients with disease behaviour B2 and B3 at diagnosis, $29.5 \%$ of initially $\mathrm{B} 1$ patients became B2 or B3 from diagnosis to five years, $43.1 \%$ from five to 10 years, $27.3 \%$ from 10 to 15 years, $10 \%$ from 15 to 20 years, and $33.3 \%$ from 20 to 25 years. These proportions were not significantly different.

Examining exclusively the change from $\mathrm{B} 2$ to B3, fewer differences were observed. Overall, 91 patients were classified as B2 at some time point in their evolution: 33 were classified as $\mathrm{B} 2$ at diagnosis and 58 were classified as B1 and became B2 over time. Among these patients the majority (78\%) remained B2 (median follow up 6.5 years (range 1-32)). In particular, in patients who were classified as B2 at diagnosis, $88 \%$ remained as B2 (median follow up 7 years $(1-30))$.

INFLUENCE OF SURGERY ON EVOLUTION OF DISEASE BEHAVIOUR

In total, 68 patients underwent surgery and the proportion of patients who had surgery over a 10 year period was $30.4 \%$ (38 of 125 patients with at least 10 years of follow up). At the time

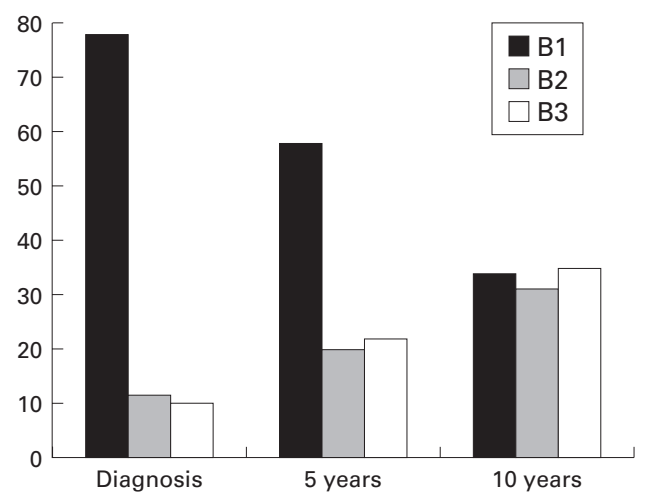

Figure 1 Evolution of disease behaviour according to the Vienna classification system over 10 years in 125 patients with Crohn's disease. The proportion of patients with non-stricturing non-penetrating (B1), stricturing (B2), and penetrating (B3) disease are shown at diagnosis, and after five and 10 years of evolution. The proportion of patients with a change in disease behaviour was highly significant over 10 years $(p<0.0001$ ) and was already significantly different from zero after one year $(p=0.04)$.

of surgery, 14 were classified as B1, 31 as B2, and 23 as B3. After surgery, 3/14 patients with a classification of $\mathrm{B} 1$ remained $\mathrm{B} 1$, eight became B2, and three became B3 (median follow up 7 years (1-20)); 24/31 patients with a classification of B2 remained B2, while seven became B3 (median follow up 8 years (1-25)).

EVOLUTION OF B AND L CLASSIFICATIONS IN A POPULATION OF 125 PATIENTS OVER 10 YEARS OF FOLLOW UP

Among these 125 patients, we had complete data on behaviour and location at all time points for 109 patients. Evolution of B and L classifications over 10 years for these 109 patients is shown in figs 1 and 2. The proportion of patients changing from B1 to B2 was $27.1 \%$, with a median duration before change of 5.5 years ( 4 months-10 years). The proportion of change from B1 to B3 was $29.4 \%$, with a median duration before change of 6 years $(1-10)$. The proportion of initially B2 patients changing from $\mathrm{B} 2$ to $\mathrm{B} 3$ was $15.4 \%$

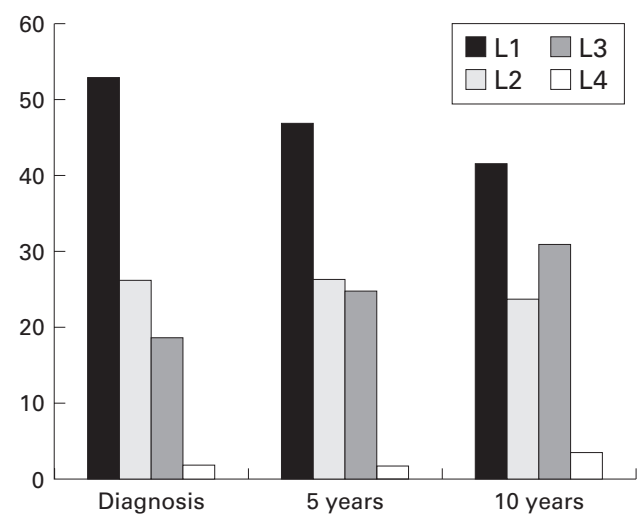

Figure 2 Evolution of disease location according to the Vienna classification system over 10 years in 125 patients with Crohn's disease. The proportion of patients with ileal (L1), colonic (L2), ileocolonic (L3), and upper gastrointestinal (L4) disease are shown at diagnosis, and after five and 10 years of evolution. The proportions of patients with a change in disease location was statistically significant over 10 years $(p<0.001)$. This proportion became significantly different from zero after five years $(p=0.01)$. 
Table 3 L (location) and B (behaviour) categories (\%) of the Vienna classification at diagnosis according to age (A). There was no significant difference between subgroups $A 1$ and $A 2$

\begin{tabular}{lcc}
\hline & A1 $(\mathrm{n}=248)$ & A2 $(\mathrm{n}=49)$ \\
\hline L1 & 43.6 & 51.1 \\
L2 & 26.1 & 29.8 \\
L3 & 25.6 & 17.0 \\
L4 & 4.7 & 2.1 \\
B1 & 75.8 & 63.8 \\
B2 & 9.5 & 17.1 \\
B3 & 14.7 & 19.1 \\
\hline
\end{tabular}

(only $2 / 13$ patients, after 3.5 and 4.5 years). Overall, after 10 years of evolution, $45.9 \%$ of patients had a change in behaviour and this proportion was already significant after one year $(3.7 \%$; $=0.04)$. Examining different types of changes in disease behaviour, the only significant change, even after 10 years, was that from B2 to B3 $(\mathrm{p}=0.15)$.

The proportion of patients with a change in disease location from L1 to another location was $20.3 \%$ (83.3\% to L3), with a median duration before change of 5.5 years $(2.3-10)$. The proportion of patients initially L2 changing to L3 or L4 was $16.7 \%$ ( $100 \%$ to L3), with a median duration before change of 7.5 years (1-10). The proportion of patients initially L3 changing to L4 was $4.8 \%(1 / 21$; change after 8.3 years). Overall, after 10 years of evolution, $15.9 \%$ had a change in location and this proportion became significant only after five years $(7.9 \% ; \mathrm{p}=0.01)$.

BL CLASSIFICATION ACCORDING TO A

A total of $248(83.5 \%)$ patients were diagnosed with Crohn's disease before 40 years of age (A1) and 49 (16.5\%) after this age (A2). The $\mathrm{BL}$ classification at diagnosis for these patients is shown in table 3. There was no significant difference between $A 1$ and $A 2$ patients. In both subgroups (A1 and A2), as in the overall population, there was a decreased proportion of patients classified as B1 and an increased proportion of $\mathrm{B} 2$ and $\mathrm{B} 3$ classifications over time, while location of disease remained more stable. The subgroup of A2 patients was too small however (only 10 patients with at least 10 years of follow up) to perform any comparison.

B CLASSIFICATION ACCORDING TO L

$\mathrm{B}$ classification according to $\mathrm{L}$ at diagnosis and after 10 years of evolution is shown in table 4 . At diagnosis, there was significantly more penetrating and less stricturing disease in pure colonic (L2) than in pure ileal (L1) locations. This was even more prominent after 10 years of evolution.

\section{Discussion}

Using the new Vienna classification of Crohn's disease in this retrospective study, we have shown striking changes in disease behaviour over the course of the disease while disease location remained relatively stable. This changing disease behaviour was significantly influenced by the location of the disease but not by age at diagnosis.

As it becomes clear that Crohn's disease represents a heterogeneous entity, ${ }^{2}$ it is of paramount importance to disclose stable and reproducible phenotypes in order to study their genetic or environmental basis. Location and behaviour of Crohn's disease are important features of the disease but their stability over the course of the disease had not been demonstrated.

We found good stability of disease location, defined according to the Vienna classification. Although the trend toward a changing pattern was statistically significant after five years of evolution, overall (over 10 years) about $85 \%$ of patients did not show a change. This relative stability has previously been suggested, even using slightly different definitions of disease location, ${ }^{5}$ although some changes may also be observed. ${ }^{22}$ During its course a disease can extend or regress within the same region and this will not change its classification according to the Vienna system. It can also regress or extend to another region. According to our results this does not seem to occur frequently. The main change we observed was from ileal (L1) or colonic (L2) to ileocolonic disease (L3). Over 10 years however it involved less than one fifth of patients with pure ileal or pure colonic disease at diagnosis. The frequency of upper gastrointestinal lesions was rare at diagnosis and few patients seemed to develop these over the course of the disease. This relative stability may be due to the retrospective character of the study and the absence of a systematic search for extension. However, in our population, median duration since the last exploration (by $x$ ray or endoscopy) of disease extent was 2.5 years suggesting that the results analysed over 10 or 25 years were probably realistic. These data indicated that disease location, according to the Vienna classification, may be determined by some stable environmental or genetic influences and that it probably represents a relevant characteristic in phenotypegenotype studies.

In contrast, we found dramatic changes in behaviour over the course of the disease. This is the first time that this changing pattern has been clearly demonstrated, despite the fact that we used a classification system considered to

Table $4 B$ (behaviour) categories (\%) of the Vienna classification according to L (location) at diagnosis and after 10 years of evolution

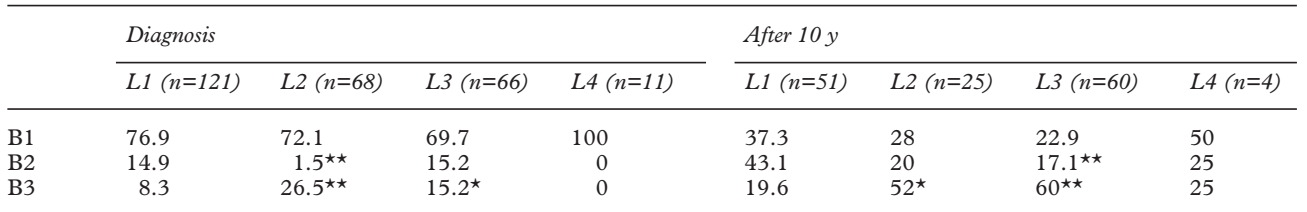

${ }^{\star} \mathrm{p}<0.05 ;{ }^{\star \star} \mathrm{p}<0.01, \mathrm{~L} 2$ or L3 compared with L1. 
have good interobserver reproducibility. ${ }^{13}$ This changing pattern over time was suggested previously in the initial publication of the Vienna classification as difference in behaviour proportions were described in populations with different durations of follow up. ${ }^{13}$ In our population, while the majority of patients had uncomplicated non-stricturing nonpenetrating disease (B1) at diagnosis, very few had uncomplicated disease after 25 years of follow up. Furthermore, the rate of change from B1 to either the stricturing (B2) or penetrating (B3) phenotype remained stable over 25 years and did not seem to decrease over time, with approximately $25-33 \%$ of B1 patients changing to $\mathrm{B} 2$ or $\mathrm{B} 3$ every five years. This suggests that in our population, if followed for long enough, virtually all patients with Crohn's disease would develop either stricturing or penetrating disease. This however may reflect a particular severity of our Crohn's disease patients in an academic referral centre. Our data did not allow us to establish a duration of the disease after which it would be unlikely for disease behaviour to change. However, when considering stricturing disease, it is striking that the majority of patients develop this pattern within 10 years and that only a minority progress to a penetrating disease. The development of a stricturing or penetrating behaviour probably represents a multifactorial phenomenon involving both environmental (including disease treatment) and genetic factors. Therefore, genetic factors may be more important in the speed at which a penetrating or stricturing behaviour develops than in the ultimate behaviour of the disease. Indeed, one could imagine that certain aspects of connective tissue metabolism are genetically determined and may influence the occurrence of stricturing or penetrating complications. ${ }^{23}$

We did not find any significant difference in disease behaviour or location in relation to age at diagnosis. In the first paper on the Vienna classification, a difference in disease location but not behaviour was found. ${ }^{13}$ This discrepancy regarding disease location may be due to the fact that we had a relatively small number of patients with a diagnosis later than 40 years of age (A2), rendering statistical analysis on this subgroup difficult. Our data also showed that the change in disease behaviour over time and relative stability of disease location were found both in $\mathrm{A} 1$ and $\mathrm{A} 2$ patients, suggesting that this changing pattern of Crohn's disease behaviour was not linked to age at diagnosis.

The final point we studied was the association between disease location and behaviour as well as the influence of disease location on disease behaviour evolution. A positive association between location and behaviour was found previously in the original description of the Vienna classification. ${ }^{13}$ As in this previous work we found an association between isolated small bowel location (L1) and stricturing disease (B2), and between colonic disease (L2) and penetrating behaviour (B3). This association was present at diagnosis and was quantitatively more prominent after 10 years. In contrast with the first paper however, in our population ileocolonic disease (L3) was also more often penetrating (B3) than stricturing (B2). This was only true after 10 years of evolution and not at diagnosis. Thus this difference from the previous study may be linked to variable duration of disease at the time of classification. The association between stricturing disease and small bowel location was previously described using different classifications. ${ }^{24}$ Apart from these previously described associations, our data also showed that changes in disease behaviour over time were noted in the various locations of the disease.

In conclusion, we have demonstrated dramatic and sustained changes in Crohn's disease behaviour over time while disease location remained more stable. Hence if location of disease, as defined by the Vienna classification, can be used in genotype-phenotype analyses, this is not the case for behaviour of the disease. Nevertheless, the rate of appearance of stricturing or penetrating complications of the disease may still be genetically determined. Therefore, for genotype-behavioural phenotype analyses, patients should be classified either after a fixed duration of disease or in categories according to the rate of appearance of stricturing or penetrating complication.

E Louis is a Research Associate at the FNRS Belgium.

1 Fiocchi C. Inflammatory bowel disease: etiology and pathogenesis. Gastroenterology 1998;115:182-205.

2 Cho JH, Brant SR. Genetics and genetic markers in inflammatory bowel disease. Curr Opin Gastroenterol 1998;14: 283-8.

3 Hugot JP, Chamaillard M, Zouali $\mathrm{H}$, et al. Association of NOD2 leucine-rich repeat variants with susceptibility to Crohn's disease. Nature 2001;411:599-603.

4 Ogura Y, Bonen DK, Inohara N, et al. A frameshift mutation in NOD2 associated with susceptibility to Crohn's disease. Nature 2001;411:603-6.

5 Sutherland LR. Different patterns of Crohn's disease. In: Prantera C, Korelitz B, eds. Crohn's disease. New York: Prantera C, Korelitz B, eds. Crohn's
Marcel Dekker, Inc., 1996:201-15.

6 Heresbach D, Alizadeh M, Bretagne JF, et al. TAP gene transporter polymorphism in inflammatory bowel diseases. Scand $\mathcal{F}$ Gastroenterol 1997;32:1022-7.

7 Plevy SE, Taylor K, De Woody KL, et al. Tumor necrosis factor (TNF) microsatellite haplotypes and perinuclear anti-neutrophil cytoplasmic antibody (pANCA) identify Crohn's disease (CD) patients with poor clinical response to anti-TNF monoclonal antibody (cA2). Gastroenterology 1997;112:A1062.

8 Bouma G, Poen AC, Garcia-Gonzalez MA, et al. HLA$\mathrm{DRB} 1{ }^{\star} 03$, but not the TNFA-308 promoter gene polymorphism, confers protection against fistulising Crohn's disease. Immunogenetics 1998;47:451-5.

9 Louis E, Peeters M, Franchimont D, et al. Tumour necrosis factor (TNF) gene polymorphism in Crohn's disease: influence on disease behaviour? Clin Exp Immunol 2000; 119:64-8.

10 Greenstein AJ, Lachman P, Sachar DB, et al. Perforating and non-perforating indications for repeated operations in Crohn's disease: evidence for two clinical forms. Gut 1988; 29:588-92.

11 Sachar DB, Adrews HA, Farmer RG, et al. Proposed classification of patient subgroups in Crohn's disease. Gastroenterol Int 1992;5:141-54.

12 Steinhart AH, Girgrah N, McLeod RS. Reliability of a Crohn's disease clinical classification scheme based on disease behavior. Inflamm Bowel Dis 1998;4:228-34.

13 Gasche C, Scholmerich J, Brynskov J, et al. A simple classification of Crohn's disease: report of the working party of the world congresses of gastroenterology,Vienna 1998. Inflamm Bowel Dis 2000;6:8-15.

14 Aeberhard P, Berchtold W, Riedtmann HJ, et al. Surgical recurrence of perforating and nonperforating Crohn's disease: a study of 101 surgically treated patients. Dis Colon Rectum 1996;39:80-7.

15 Lautenbach E, Berlin JA, Lichtenstein GR. Risk factors for early postoperative recurrence of Crohn's disease. Gastroenterology 1998;115:259-67. 
16 Yamamoto T, Allan RN, Keighley MR. Perforating ileocecal Crohn's disease does not carry a high risk of recurrence but usually re-presents as perforating disease. Dis Colon Rectun 1999;42:519-24.

17 Peeters M, Nevens H, Baert F, et al. Familial aggregation in Crohn's disease: increased age-adjusted risk and concordance in clinical characteristics. Gastroenterology 1996;111: 597-603.

18 Bayless TM, Tokayer AZ, Polito JM II, et al. Crohn's disease: concordance for site and clinical type in affected family members-potential hereditary influences. Gastroenterology 1996;111:573-9.

19 Satsangi J, Grootscholten C, Holt $\mathrm{H}$, et al. Clinical patterns of familial inflammatory bowel disease. Gut 1996;38:738-41.

20 Vasiliauskas EA, Plevy SE, Landers CJ, et al. Perinuclear antineutrophil cytoplasmic antibodies in patients with
Crohn's disease define a clinical subgroup. Gastroenterology 1996;110:1810-19.

21 Satsangi J, Landers CJ, Welsh KI, et al. The presence of anti-neutrophil antibodies reflects clinical and genetic heterogeneity within inflammatory bowel disease. Inflamm Bowel Dis 1998;4:18-26.

22 Peschard S, Beaugerie L, Carbonnel F, et al. La maladie de Crohn iléale pure reste-t-elle toujours localisée ? Gastroenterol Clin Biol 1998;22:A58.

23 Gilberts EC, Greenstein AJ, Katsel P, et al. Molecular evidence for two forms of Crohn's disease. Proc Natl Acad Sci USA 1994;91:12721-4.

24 Farmer RG, Hawk WA, Turnbull RB. Indications for surgery in Crohn's disease. An analysis of 500 cases. Gastroenterology 1976;71:245-50. 